\title{
24
}

\section{Experiences with concurrent TTCN for GSM/SS7 testing}

Elyane Prevot ${ }^{1}$, Michel $D^{\prime}$ Hooge $^{2}$, Patrice Labbe ${ }^{2}$

1 France Télécom - CNET

38-40 avenue du Général Leclerc, 92131 Issy-Les-Moulineaux

E-mail : bruno.chatras@issy.cnet.fr

Tel : +33 1 45-29-58-53 Fax : +33 1 46-29-31-42

2 Trialog

25 rue du Général Foy, 75008 Paris

E-mail:100411.2233@compuserve.com

Tel : +33 1 44-70-61-00 Fax : +33 142-94-80-64

\begin{abstract}
Global System for Mobile communication (GSM) is a European standard which defines telecommunication services for mobile subscribers. At the end of 1995, the European Telecommunication Standard Institute (ETSI) released the Mobile Application Part (MAP) version 2 protocol which supports signalling between GSM phase 2 network entities. It also provided the corresponding conformance test purpose specification.

The testing of this protocol involved numerous interfaces and thus required multi-party testing.

The introduction of concurrent TTCN reduced the testing specification complexity.

This paper describes a practical experience with concurrent TTCN in the GSM environment. It demonstrates the importance of completeness in test purpose specification and the advantage of allocating substantial resources to both ATS development and ATS validation to ensure ATS quality. It also presents a TTCN style guide in order to improve maintenance tasks. It describes some TTCN deficiencies and proposes enhancements.
\end{abstract}

Conformance Testing, GSM, TTCN

Keywords 


\section{INTRODUCTION}

The Global System for Mobile communication (GSM) is a European standard for digital cellular systems which defines telecommunication services for mobile subscribers. Numerous operators have implemented this standard in many countries and provide the so-called GSM pan-European service.

The first version of this standard (known as GSM phase 1) was officially released in 1991 by ETSI, the European Standardisation Institute for Telecommunication. Currently available implementations are still based on this initial version. However, since then ETSI has defined GSM phase 2 by enhancing the GSM standard to provide additional services.

Since it is unlikely that all GSM phase 1 operators will upgrade their network entities at the same time, interoperability between GSM network entities of different phases is a strong requirement to ensure successful network operation from a subscriber's point of view. Conformance testing of the GSM protocols is the best way to increase the likelihood that mixed networks can interoperate to provide the best quality of service to GSM subscribers.

This paper presents experiences in the conformance test specification of the GSM phase 2 Mobile Application Part protocol (MAP). The introduction describes briefly the GSM network architecture and protocol stack. It also presents the base standard, MAP, to give an idea of the characteristics of the tested protocol. Section 2 discusses different aspects of test specification. Sections 3 and 4 describe the ATS and ETS production as well as our practical experience with concurrent TTCN. A comparison with GSM phase 1 testing is also given. The conclusion gives an overview of the man-power involved. A glossary of acronyms is provided at the end of the article.

\subsection{GSM Network}

GSM provides mobility and roaming facilities that enable subscribers to receive or send calls regardless of their location [Mouly]. The GSM network architecture and associated protocols are shown in Figure 1.

The ability to call a mobile subscriber at any time and anywhere in Europe implies the knowledge of its location in real-time. The Home Location Register (HLR), is an "intelligent" data base which stores the user's subscription and current location. This register is then interrogated by a Mobile service Switching Centre (MSC) each time a call is destined to a given subscriber. In other words, the HLR is able to provide routing information to transfer the incoming call to the called subscriber.

In order to locate a subscriber, the GSM service area is divided into elementary areas (cells) which in turn are logically grouped in location areas. MSCs associated with data bases, known as Visited Location Registers (VLR), manage those location areas. Thus, when a mobile subscriber moves from one area to another, it informs the MSC/VLR in charge of this area. The MSC/VLR will then inform the HLR of the current location of the subscriber.

The MSC/VLR also provides to roaming subscribers call related GSM services such as outgoing call handling, incoming call delivery, short message delivery, and supplementary services. Subscribers give their location to the network or ask for GSM telecommunication services via a Base Station Sub-system (BSS) by using radio access. 


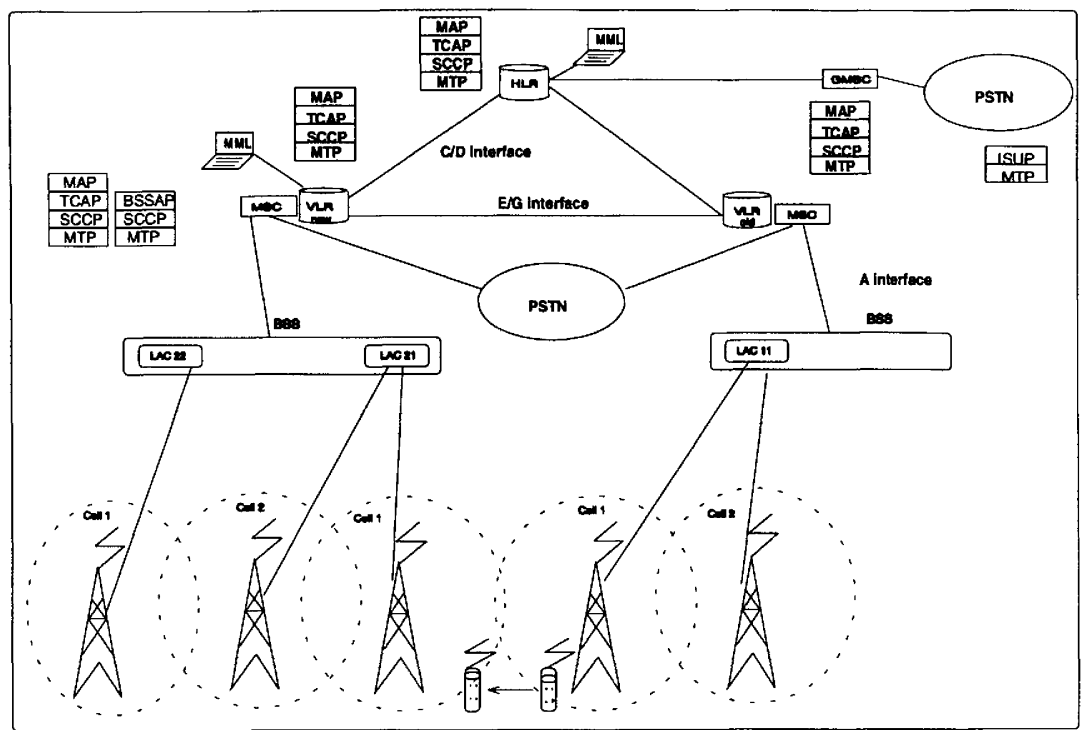

Figure 1: GSM network.

\subsection{Base Specification: MAP}

The Mobile Application Part (MAP) protocol supports the signalling between the GSM network entities, with the exception of the MSC/VLR which is supported by the Base Station Application Part protocol (BSSAP). MAP makes use of the Transaction Capabilities of the Signalling System $\mathrm{N}^{0} 7$ protocol stack [Webster].

MAP is a complex protocol which makes use of 46 messages, described in ASN.1, each of which is used by MAP procedures to realise a given functionality. For instance, the procedure "Location Updating", which takes place between the VLR and the HLR in order to register the current subscriber location, involves the exchange of 6 messages: the request and acknowledgement of UpdateLocation, InsertSubscriberData, and ActivateTraceMode.

MAP version 1 provides the GSM phase 1 services while MAP version 2 supports the GSM phase 2 services. MAP version 2 also corrects some deficiencies in MAP version 1 . It was frozen and published at the end of 1995 .

One of the main GSM phase 2 characteristics is to allow a GSM phase 1 entity to interoperate with a GSM phase 2 entity. The objective of this compatibility is to allow soft migration from GSM phase 1 to GSM phase 2 by providing GSM operators with the option of implementing only part of GSM phase 2 functionalities. Thus the compatibility between versions has to be checked for GSM phase 2 entities. This imposes strong requirements on the MAP version 2 test specification. 


\section{TEST SPECIFICATION}

GSM equipment suppliers are in the process of implementing GSM phase 2. Thus, there is a need for conformance testing of the GSM phase 2 network entities to ensure successful network operation. As we can see from Figure 1, testing the MAP protocol is a complex task due to the number of interfaces involved and the diversity of protocol used in those interfaces. This means that the MAP conformance testing of every GSM network entity is a big task, and it is often said that suppliers and operators are reluctant to pay for it. Thus in order to ensure conformance testing, consortia were created, such as the MoU/CONIG for the MAP version 1 conformance testing, and ETSI work items were planned.

ETSI published the first stable draft of the MAP version 2 test specification at the beginning of 1995. Due to lack of resources and TTCN expertise, ETSI defined only the test purposes. It was planned that the ATS specification in TTCN be done by an external body, such as an ETSI Project Team or a CTS project. The idea was to provide the test purposes as input to this external body with the support of ETSI to solve open issues on MAP protocol. Unfortunately, no funds were allocated for the derivation of the TTCN ATS from the ETSI test purposes. Thus France Télécom-CNET decided to develop the TTCN ATS on its own by using the services of Trialog, an external software house.

This project started with the definition of the ATS specification methodology and the analysis of all possible problems by taking a sample of representative test purposes. It then continued with the writing of all test cases. Section 3 gives an overview of the ATS development methodology. GSM phase 2 testing is based on the phase 1 experiences [Berkhout, Koster]

\subsection{Test Purposes}

The MAP version 1 conformance test specification was a set of 1000 tests. ETSI produced around 1600 test purposes for the MAP version 2 conformance test specification, the increase in number coming mainly from additional functionalities introduced in GSM phase 2. Table 1 gives an overview of number of test purposes allocated per procedure.

Table 1: Test purposes allocation

\begin{tabular}{lllll}
\hline & \multicolumn{2}{l}{ Valid Behaviour Tests } & \multicolumn{2}{l}{ Invalid Behaviour Tests } \\
\hline & Number & \multicolumn{3}{l}{ Number } \\
\hline Inter-Working between versions & 57 & $(90 \%)$ & 6 & $(10 \%)$ \\
Operation and Maintenance & 166 & $(73 \%)$ & 62 & $(27 \%)$ \\
Location Management & 159 & $(77 \%)$ & 44 & $(23 \%)$ \\
Call Handling & 301 & $(96 \%)$ & 11 & $(4 \%)$ \\
Handover & 294 & $(71 \%)$ & 120 & $(29 \%)$ \\
Supplementary Services & 93 & $(71 \%)$ & 38 & $(29 \%)$ \\
Short Messages & 150 & $(72 \%)$ & 57 & $(28 \%)$ \\
\hline
\end{tabular}

ETSI based the MAP version 2 conformance test specification on the MAP version 1 test specification output. Enhancements was made by increasing the number of values tested in message fields, and syntactical error checking was reduced to the most significant cases. 
The test suite structure was modified to be entity oriented instead of procedure oriented. This calls for a test suite structure with one group per GSM entity, i.e. HLR, MSC/VLR, and SMS-GMSC/IWMSC (short message service for the gateway and interworking MSCs). Then every entity group is sub-divided into several groups, each of which is related to one procedure (e.g. location management, call handling ). Every procedure group is divided as well into two sub-groups: valid behaviour testing and invalid behaviour testing. Each of these is sub-divided in turn to take care of several testing aspects such as data variation, structure variation, and syntax errors.

\begin{tabular}{|c|c|c|}
\hline IUT : & \multicolumn{2}{|l|}{ HLR } \\
\hline Test Name : & \multicolumn{2}{|l|}{ LMLU_1 } \\
\hline Test Identification : & \multicolumn{2}{|c|}{ Location Update with Insert Subscriber Data. } \\
\hline Test Purpose : & \multicolumn{2}{|c|}{$\begin{array}{l}\text { On receipt of an Update Location Request from the MSC/VLR with the } \\
\text { default parameters, check that the IUT } \\
\text { - continues the dialogue towards the MSC/VLR and sends an } \\
\text { InsertSubscriberData invocation, } \\
\text { - ends the Update Location request from the MSC/VLR and returns a positive } \\
\text { result to the Update Location Request. }\end{array}$} \\
\hline Pre-condition & \multicolumn{2}{|c|}{ ProfileLM1, IUT in normal operation } \\
\hline $\begin{array}{l}\text { Reference to flow } \\
\text { diagram: }\end{array}$ & \multicolumn{2}{|l|}{ Section 16.1.1.4. } \\
\hline \multicolumn{2}{|c|}{ STIMULUS } & RESPONSE \\
\hline \multicolumn{2}{|c|}{$\begin{array}{l}\text { 1. } \\
\text { UpdateLocation.argument. } \\
\text { Imsi = Identity.imsi, } \\
\text { lmsi = ( not present }) \\
\text { locationInfo.mscNumber = PICS/PIXIT for } \\
\text { MSCNumber 1, } \\
\text { vlr-Number = PICS/PIXIT for VLRNumber } 1 . \\
\text { 3. } \\
\text { (If current Location Info in IUT is different to } \\
\text { UpdateLocation.argument.vlr-Number):- } \\
\text { CancelLocation.result }\end{array}$} & $\begin{array}{l}2 . \\
\text { (If current Location Info in IUT is different to } \\
\text { UpdateLocation.argument.vlr-Number):- } \\
\text { CancelLocation.argument } \\
\text { imsi = Identity.imsi } \\
\text { 4. } \\
\text { InsertSubscriberData.argument. } \\
\text { imsi = (not present) } \\
\text { subscriberdata. } \\
\text { msisdn = Identity.msisdn, } \\
\text { category = Ordinary Subscriber, } \\
\text { subscriber status = service granted, } \\
\text { bearerServiceList = (not present), } \\
\text { teleServiceList. } \\
\text { teleServiceCode = telephony } \\
\text { provisionedSS = (not present), } \\
\text { odb-Data = (not present). } \\
\text { roamingRestrictionDueToUnsupportedFeature = } \\
\text { (not present) } \\
\text { regionalSubscriptionData = (not present) }\end{array}$ \\
\hline \multicolumn{2}{|c|}{$\begin{array}{l}\text { InsertSubscriberData.result } \\
\text { teleserviceList }=\text { (not present) } \\
\text { bearerServiceList }=\text { (not present }) \\
\text { ss-List }=\text { (not present }) \\
\text { odb-GeneralData }=\text { (not present) } \\
\text { regionalSubscriptionResponse }=\text { (not present) }\end{array}$} & $\begin{array}{l}\text { 6. } \\
\text { UpdateLocation.result. } \\
\text { extensibleUpdateLocationRes }\{ \\
\text { hlr-Number = Profile.HLRNumber । }\end{array}$ \\
\hline
\end{tabular}

Figure 2: Test purpose specification example. 
The MAP version 1 test purposes were defined using a prose description. Although the writers were TTCN experts rather than MAP experts, the final product was of medium quality from both a TTCN and MAP point of view. The TTCN errors were essentially due to the fact that no TTCN editor tools or compilers were available at that time, so the test cases were written directly in MP format.

For phase 2, ETSI decided to define the test objectives in a way that gives enough information to make the TTCN specification easier since it is likely that the ATS specifiers will not be GSM protocol experts. The test purposes have thus been formalised in such a way that every Stimulus (tester behaviour) and every Response (IUT behaviour) are explicitly given. The Stimulus and Response messages are described to look like ASN.1 value notation. Figure 2 gives an example of this type of test purpose specification.

The Preamble and Postamble are implicitly specified by giving the required IUT status in terms of subscriber profile (e.g. roaming restrictions, supplementary services, location information). A table which defines the default Stimulus and Response message contents is at the beginning of each section in order to improve the readability of the specification.

\subsection{Test Architecture}

Previous work on GSM Mobile Network System testing was limited by the impossibility of using a multi-party abstract test method and concurrent TTCN [Koster], [Ahtiainen], [Berkhout]. This project had the good fortune to start when the concurrent standard and tools became available. The test architecture, i.e. the test method, of this project was defined following ISO 9646 recommendation [ISO9646]. The chosen method is "remote" and "multiparty".

The "remote" aspect comes from the fact that the SUT (System Under Test) is in its operational configuration, so it is not possible to access directly the upper and lower bounds of the IUT. As a consequence, the UT (Upper Tester) is in the SUT while the LT (Lower Tester) is in the test system. The later interacts with the IUT "remotely", i.e. through indirect ways that should have the same effect on the IUT as direct actions. For example, in order to call a procedure belonging to the MAP upper interface, the operator is asked to enter a Man Machine Language (MML) command which calls the procedures to be tested.

The "multi-party" aspect is due to the fact that some of the GSM entities in which MAP protocol is implemented have numerous interfaces. As shown in Figure 3, the MSC/VLR has interfaces with the HLR (MAP), the base station (BSSMAP or DTAP), the fixed telephone network (ISUP), and the operator (MML), among others. A PCO (point of control and observation) must be defined for each interface. The LT must deal with all these interfaces. This requires the use of the "multi-party" test method in conjunction with Concurrent TTCN, which are defined in the first Amendment to ISO 9646 and explained below.

\section{ATS PRODUCTION/DERIVATION}

In order to be as close as possible to the test suite structure presented in section 2.1, one ATS per entity was produced. Each ATS structure follows the test suite structure defined by ETSI. 


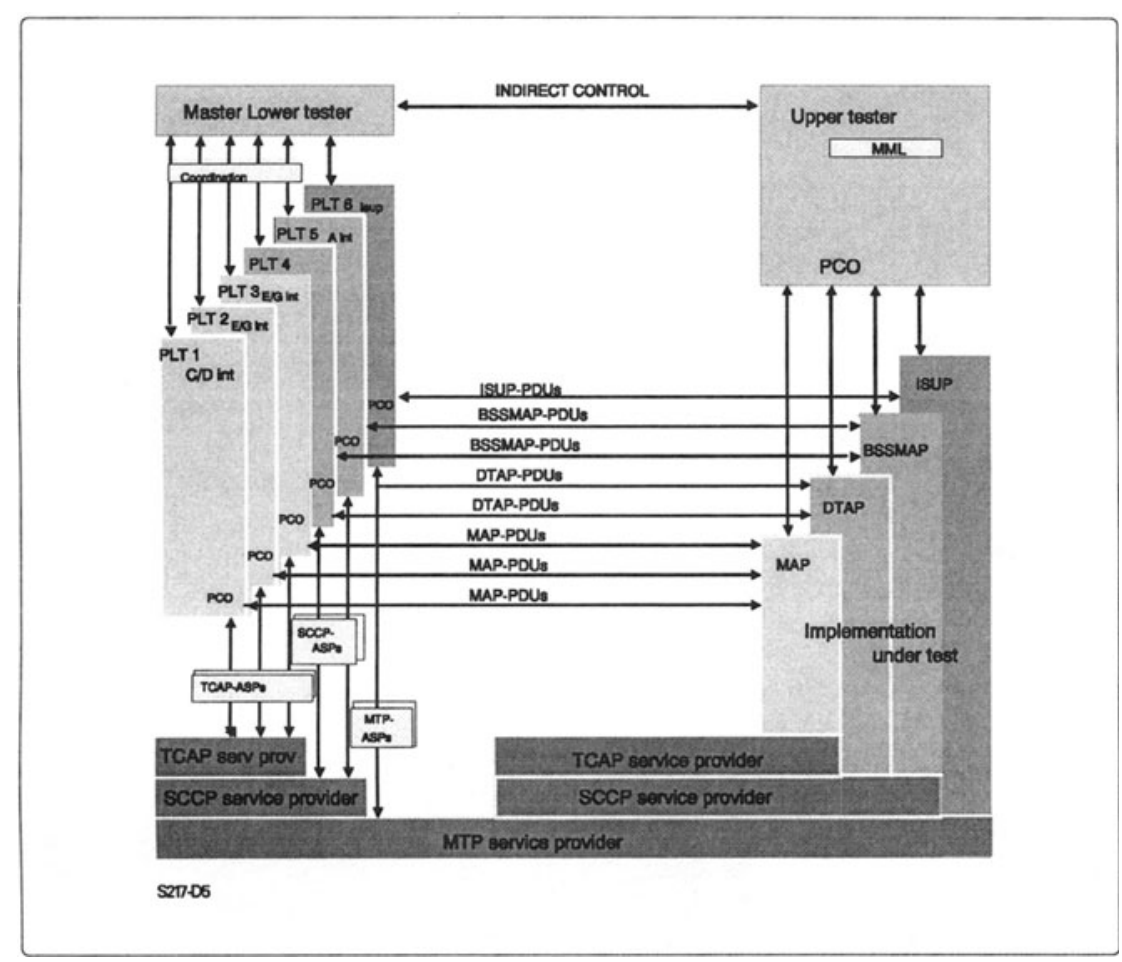

Figure 3: Multi-party testing of MSC/VLR.

\subsection{Concurrent TTCN}

As shown in the previous section, in order to validate a MAP implementation, it is necessary to examine simultaneously the PCOs located at all the interfaces. If we use the IS version of TTCN, which does not support concurrency, each test case must take into account all the messages on all the interfaces at the same time [Walter 92]. This leads to very complex test cases.

With concurrent TTCN, it is possible to define the messages sent or received separately on each interface and to describe the synchronisation between them. Since a new version of the TTCN compiler is available with concurrent TTCN facilities, it was possible to take advantage of these new facilities in this project.

\subsection{TTCN Style Guide}

Since a test suite is written by one group of people and often debugged or maintained by another, it is very important to have clear and self-explanatory code. To meet this goal, a style guide was developed for this project to simplify the debugging and maintenance tasks. This system proved to be very successful. If the same had been done for MAP version 1 test 
specification, maintenance work would had been facilitated and a number of resources would had been saved.

\section{Test Suite Fragmentation}

Although the tabular form of TTCN divides a test suite into convenient tables, the machine processable form is a giant single file for an entire test suite. While developing the MP form of the test suite, a hierarchy of directories and groups of small files were used to divide the suite into more manageable fragments. A file containing the test suite skeleton uses include directives to incorporate the MP code stored in other files.

\section{Naming Convention}

The EWOS/TA/93/006 [EWOS] explains the importance of naming conventions and gives guidelines on this point. However, the examples given use underscores, which lengthen names, and the names are mostly in uppercase, which lessens readability. Therefore we developed naming conventions which better suit our needs.

A strict and exhaustive naming convention was developed to ease the naming and the reading of identifiers for each test element, ranging from simple constants to test steps. Each identifier is divided into 2 or 3 semantically different parts. The following rules describe the basis of these conventions.

A prefix identifies the type of the identifier and its domain of application. For example, $v$ stands for variable, $c$ for constant, $p x$ for pixit and so on. This is useful for recognising the function of an identifier where a number of different types could be used. This is followed by a short, usually abbreviated, but easily understandable description of the function. For example, VlrAddr stands for Visitor Location Register Address. A postfix can be added to distinguish between identifiers which would be identical when using only the first two fields of the naming convention. Capital letters are used at the start of each part as separators. However, an underscore can be used to separate parts to improve readability.
pxIutAddrMsc
pxIutAddrGmsc
a pixit for the IUT address for the MSC mode.
a pixit for the IUT address for the GMSC mode.

Special conventions apply to constraint identifiers since they represent a large part of the test suite. The first character of the prefix defines whether it is used in an indication or request. This is useful since the syntax of the two types of constraint is different. The protocol used is also part of the prefix, where $M$ stands for MAP, D for DTAP, etc.
qM_ATMa_Omcld
request, MAP, Activate Trace Mode Argument, OMC Id
iM_ISDr_ss field included.
iD_LocUpdAcc_noTmsi indication, MAP, Insert Subscriber Data Result, Supplementary Service field included. indication, DTAP, Location Updating Accept, no TMSI field.

For test steps, the prefix contains the name of the entity simulated, and if applicable the role played.
MsclAnchPerformHO
Entity = Msc \#1; Role = Anchor MSC; Service = Perform Handover
HIr1DSD_UError
Entity $=$ Hlr \#1; Role $=$ N/A; Service $=$ Delete Subscriber Data; Specialisation $=$ Waiting for a negative result. 


\section{Local Tree Hierarchy}

In order to see the basic structure of a test case at a glance, each PTC (Parallel Test Component) is represented as a local tree in the case and not as a test step. Each of these local trees is in turn divided into three local trees which represent the preamble, the body, and the postamble. Within these trees, test steps can be called. In this way, the creation of many test steps which cannot be reused is avoided. Figure 4 shows an example of a concurrent TTCN test case.

\subsection{Suggestions for TTCN Improvements}

During our experience in writing test suites in TTCN, we encountered certain difficulties with the language. The concurrent aspects of TTCN are useful and pose no problems, but often the basic TTCN syntax is awkward. The following sections describe our suggestions for improvements in basic TTCN that would make programming easier.

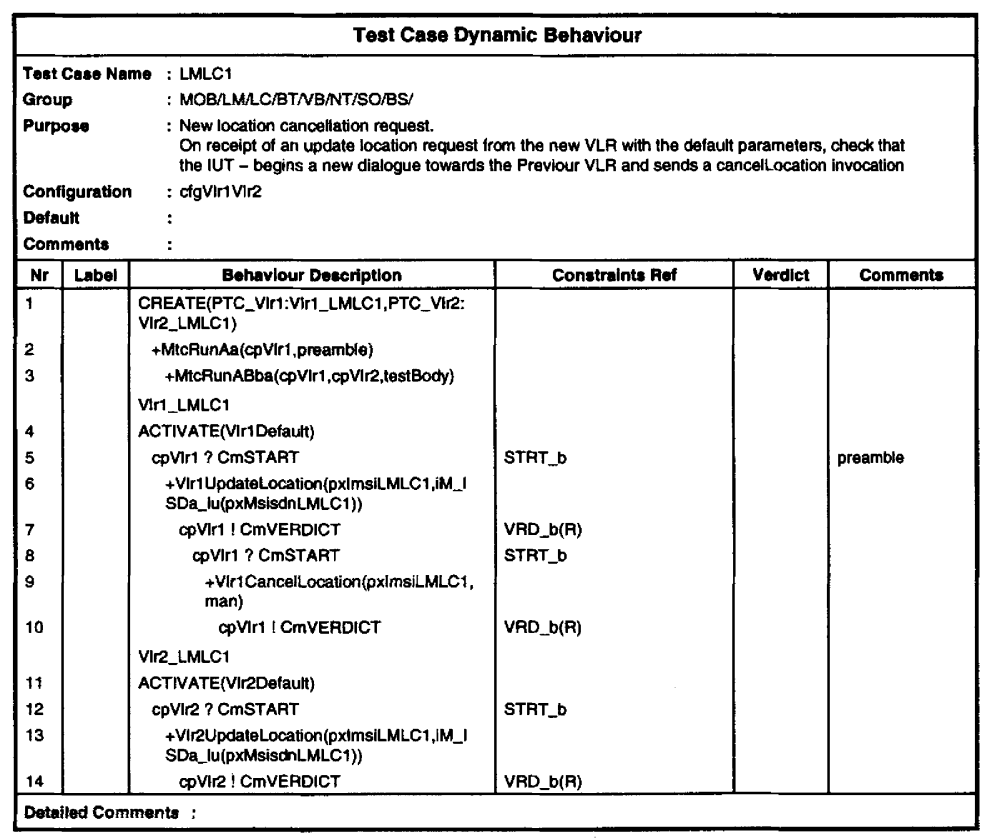

Figure 4: Example of Concurrent TTCN test case. 


\section{Lack of Modularity}

One of the more important limitations of the TTCN language comes from the fact that an Abstract Test Suite is defined, following ISO 9646-3, as a single, monolithic program. An ATS is defined as a sequence of declarations, test cases, test steps, and so on. Although each concept is defined separately, for a big test suite this kind of structure is neither sufficient or efficient. Tools have been developed to get around this aspect, with the possibility of navigating easily in a large test suite, but it would seem much better to improve the language in order to introduce new modular capabilities.

Most programming languages allow a program to be divided into modules, such as files and procedures. When developing a test suite, it would be much better to be able to deal with "modules" or "sub-suites", each one dealing with one aspect of the IUT to test. Declarations and tests steps defined in other modules could be declared as "external". Before test execution, all these modules would be linked together in order to produce the complete ETS.

Furthermore, is it impossible at this stage to think of an "Object TTCN", in order to climb two evolutionary steps of software technology at a time? TTCN could benefit a great deal from object oriented aspects such as static independence between test suite components, declarations with classes and object instances, inheritance mechanisms, and dynamic communication between PTCs and MTCs (Master Test Components).

\section{Timer as a Parameter}

Currently in TTCN it is not possible to pass a timer as a parameter to a step, a default, or a local tree. As a result, if a step is to be used with two different timers, one solution is to duplicate the step with the timer as the only difference. Another is to pass a variable as a parameter which identifies the timer to use, and the choice of which behaviour line to use is based on the value of the variable. It would simplify the code if a timer could be passed as a parameter.

\section{Select Expression to Parameterize Constraints}

Because constraints cannot be parameterized with a select-expression, we made the selection in the dynamic part by qualifying the two alternate receive events accordingly. Since it is known before run-time which one is expected, it would be conceptually cleaner and practically more concise to have the following type of construct:

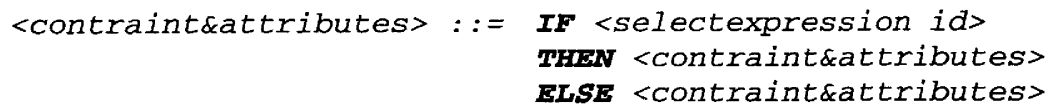

\section{Qualified Attachment}

Qualifying a step would be convenient and concise. The format could be as follows:

$$
+T[Q]
$$


The qualifier $Q$ would be ANDed to the qualifiers of first column events when the step is expanded. Non qualified events would be considered to have an implicit constant qualifier [TRUE]. Qualifying steps with non-qualifiable events (e.g. GOTO ) in the first column would lead either to a syntax error or to a different policy. For instance:

$$
+T[Q]
$$

would be equivalent to :

$$
\begin{aligned}
& \text { [Q] } \\
& +T
\end{aligned}
$$

when $\mathrm{T}$ has non-qualifiable events in the first column.

\section{Optional Step Attachment}

Some test cases would be easier to write if test steps could be made optional. In the following example, brackets indicate an optional step.

$$
\begin{array}{ll}
+T 1 & +T 2 \\
+T 2
\end{array}
$$

would be equivalent to :

$$
\begin{aligned}
& +[T 1] \\
& \\
& +T 2
\end{aligned}
$$

The example given is rather simple, but complexity increases exponentially when the sequence of optional steps is longer. An alternative would be to encapsulate all that follows an optional step into an intermediate tree, but this leads to deep encapsulation and poor readability.

\subsection{Practical Experience}

ATS derivation was carried out by a team of 3 people over approximately 11 months. The time necessary to produce the ATS is shown in the following table. This does not include time for tasks such as management, "peripheral" activities like methodology definition, or quality control.

The first test case family is a special case, because it covers all MAP protocol aspects for GSM $\mathrm{v} 1$ and $\mathrm{v} 2$, and the productivity is not representative for standard test case derivation. The two other families, with a production rate of 2.4 to 3 test cases per day, are a more reliable basis to use to determine the time needed for ATS derivation.

It is important to note that the times below include all operations related to ATS derivation: test purpose and protocol analysis, TTCN writing, syntactical adjustment after compilation, technical review, and debugging after ETS execution. Thus it not the time needed simply to write a suite in TTCN. 
Table 2: Time necessary to produce the ATS

\begin{tabular}{llll}
\hline Test case families & $\begin{array}{l}\text { Number of } \\
\text { test cases }\end{array}$ & Man/days & Test per day \\
\hline Inter-Working & 63 & 75 & 0.84 \\
Operation and Maintenance & 228 & 78 & 2.9 \\
Location Management & 203 & 81 & 2.5 \\
Call Handling Procedure & 312 & 103 & 3 \\
Handover & 414 & 173 & 2.4 \\
Supplementary Services & 131 & 50 & 2.8 \\
Short Messages & 207 & 72 & 2.9 \\
TOTAL & 1558 & 630 & 2.5 \\
\hline
\end{tabular}

\section{ETS GENERATION AND VALIDATION}

The Executable Test Suite (ETS) generation and validation consisted of running TTCN test cases first with a simulated IUT and then a real one. The chosen tester was the Tekelec Octopus tool which provides both ETS development and execution environments.

\subsection{Test Tool}

Octopus is a test execution software environment which provides the means to produce ETS from TTCN ATS in a quasi-automatic way [Ahtiainen]. The production is automated when the protocol is defined with the ASN.1 notation. This is the case for the MAP protocol. Octopus also implements concurrent TTCN defined in TTCN-IS 9646-3, AM1. This environment was mainly developed for SS7 testing, but other signalling protocols (Internet, OSI, etc.) can be tested with a minimum software development. Octopus provides three main tools.

- A tool to derive ETS from ATS which is called a "TTCN to C compiler ".

- A tool to configure the underlying protocol stack used by the test suite, which is the protocol stack beyond the different test suite PCOs.

- A tool to execute an ETS through a graphical user interface. This tool provides tracing, pics/pixit definition, and test case selection facilities.

\subsection{Generation}

One ATS per GSM network entity was defined, so three ETSs were produced. ETS generation comprised several steps on the Octopus platform.

1. The first step was to generate the executable $C$ code files.

2. The second step was to interface the test suite and the underlying protocol stack by mapping the ATS Abstract Service Primitives to the underlying protocol stack primitives. 
3. The third test step was to write the $\mathrm{C}$ code functions for the test suite operation. A number of test suite operations were defined, which involved a good deal programming effort. This was necessary mainly for the MSC/VLR test suite because the structure of information elements of the BSSAP protocol is different from MAP's even though they both carry the same information. Thus mapping functions were needed. This issue shows that a harmonisation of interworking protocols would facilitate the testing process.

4. The last step consisted of setting PIXIT and PICS parameters and the test configuration. A great number of PICS/PIXITs were defined due to the protocol's complexity and thus this step required substantial effort. For instance the HLR test suite requires the creation of different types of subscriber profiles in order to make data variation of the MAP InsertSubscriberData message. If the purpose of a test case is to modify some HLR data then a different subscriber type in the HLR data base is needed for each test case. Indeed, since a test case execution verdict is unsure, the HLR data base status at the end of the test case is also unsure. A postamble can be defined to put the HLR back in the initial state, but it is uncertain what goes wrong within the test case if it fails. Thus, we avoid this uncertain solution. This is one big problem of protocol conformance testing which involves data bases.

\subsection{ETS Validation}

The ATS validation is often skipped when developed within an official project such as an ETSI or CTS project, because there is no volunteer or no allocated funding for this task. However, in order to ensure a high quality ATS, this project dedicated some resources to this task. Since it is not easy to review an ATS only by making reading review, this task is often postponed to the ETS validation. We followed three approaches to validate an ETS.

- The first one was to make a reading review of ATS TTCN test cases while waiting for the availability of a real IUT or a reference implementation.

- The second way was to simulate the IUT within the tester by using the Octopus simulation facility. This simulation requires development of another test suite to play the role of the IUT. The test cases of this test suite provide a kind of simplified reference implementation but are more than a symmetric realisation of the MAP test suites.

- The third approach was to run some test cases against a real MAP entity on a platform. The IUT was in fact a partial implementation of the MAP version 2 protocol. Thus, only a few capabilities were available and thus testable.

It was not realistic to evaluate 1600 test cases, so a different strategy was adopted. A sample of representative test cases was selected to have a good coverage of the test suite. The ETS validation was performed in parallel with the ATS development. The ATS development was made procedure by procedure for each entity or test suite. This approach allowed the evaluation of test cases related to one procedure while test cases of another procedure were being specified. In this way, the evaluation process was optimised. It started when the first set of test cases related to one procedure were available, which was about two months after the start of the project. It ended one month after the delivery of the last set of test cases.

This evaluation and debug tasks costs around 5 man-months for the three test suites of 1600 tests. Table 3 gives an overview of resources dedicated to every ETS evaluation method mentioned above. 
Table3: ETS evaluation man power

\begin{tabular}{lll}
\hline & $H \boldsymbol{H}$ & $M S C N L R$ \\
\hline Reading review & 3 man-weeks & 7 man-weeks \\
IUT simulation & 3 man-weeks & 5 man-weeks \\
Real IUT & 1 man-week & 1 man-week \\
\hline
\end{tabular}

Test suite evaluation leads to frequent modifications of the TTCN ATS, which requires several compilations. Since an ATS is seen as a whole unit, no partial compilation can be made. This highlights a practical deficiency in TTCN. It would be useful if the tester or the TTCN editor could provide a tool which extracts either a test case or a group of test cases and the TTCN components used to generate the equivalent MP. The support of this functionality in the Octopus environment is under study.

\section{CONCLUSION}

The GSM MAP phase 2 protocol conformance testing specification was an extended project and involved numerous resources. Around 35 man-months were allocated to the production and validation of 1600 TTCN test cases.The project proved that the detailed ETSI test purpose specification substantially facilitated test case specifier's task.

This experience shows that the introduction of the concurrent TTCN of the 9646-3 standard simplified and improved the readibility of TTCN ATS specification. The dedication of resources to the TTCN programing style at the very early stage of the project ensured the simplification of debugging and later maintenance tasks.

Finally, even though the application of concurrent TTCN to a huge ATS highlights some TTCN deficiencies, this amendment fulfilled most of our requirements. Therefore, we encourage the use of TTCN as a conformance test specification language and we hope ISO will continue to enhance this standard to answer the needs of test specifiers.

\section{ACKNOWLEDGMENTS}

The authors would like to thank their colleagues Barbara Raither, Vincent Belaiche (Trialog), and Bruno Chatras (France Telecom - CNET) for their useful comments during the elaboration of this paper.

\section{REFERENCES}

A.Ahtiainen, B. Chatras, M. Hornbeek, S. Kesti (1993). Experiences with Octopus automated TTCN translation tools applied to GSM/SS7, in Proceedings of IWPTS 6th (ed.O.Rafiq).

S.R. Berkhout, M.F. Witteman (1991). Application of multi-party conformance test methods to the GSM mobile network system, in Proceedings of IWPTS 3rd (ed. I. Davidson and D.M.Litwack), Elsevier Science Publishers, IFIP 91.

EWOS (1993). EWOS/TA/93/006 : The TTCN Style Guide and Quality Criteria, 
ITU (1994). ITU-T Recommendation X.290 to X.296: OSI Conformance testing methodology and framework for protocol recommandations for ITU-T applications (Also published as ISO/IEC 9646).

ISO Test Methods and their Applicability to the GSM Mobile Network System, Rüdiger Köster, Markus Dicks, DETECOM

ETSI SMG. ETS-300.599 : European digital cellular telecommunications system (Phase 2). Mobile Application Part (MAP) specification (GSM 09.02).

M.Mouly, M.B.Pautet (1992). The GSM System for Mobile Communications. Michel Mouly and Marie-Bernadette Pautet Editors.

T.Walter and B.Plattner (1992). An operational semantics for concurrent TTCN in Proceeding of IWPTS 5 th (ed. G.v Bochman, R.Dssouli and A.Das).

T.Walter and F.Kristoffersen (1994). TTCN test case correctness validation, in Proceedings of IWPTS 7th.

N.Webster (1995). The Testing of BT's Intelligent Peripheral using abstract test suite from ETSI, in Proceedings of IWPTS 8th (ed. A.Cavalli and S.Budkowski).

\section{BIOGRAPHY}

Elyane Prévot graduated from ENSSAT in 1988. She has been involved in the standardization and implementation of test specifications for GSM networks since 1990 when she joined France Telecom - CNET. From 1994 to 1995 she chaired the ETSI ad-hoc group on Mobile Application Part test specification for GSM phase 2.

Michel d'Hooge graduated from Supelec in 1993. Since then he has been a software development specialist at Trialog. He was involved in this project since its conception.

Patrice Labbé is a real-time software and network expert who has more than 15 years of professional experience. He has been involved in numerous avionics, automotive, and telecom projects, often for methodological or test/validation purposes.

\section{GLOSSARY}

$\begin{array}{ll}\text { ASN.1 } & \text { Abstract Syntax Notation one } \\ \text { ATS } & \text { Abstract Test Suite } \\ \text { BSS } & \text { Base Station Sub-system } \\ \text { BSSAP } & \text { Base Station Sub-system Application Part } \\ \text { BSSMAP } & \text { BSS Management Part } \\ \text { DTAP } & \text { Direct Transfer Part } \\ \text { ETS } & \text { Executable Test Suite } \\ \text { ETSI } & \text { European Telecommunication Standard Institute } \\ \text { GSM } & \text { Global System for Mobile communication } \\ \text { HLR } & \text { Home Location Register } \\ \text { ISUP } & \text { ISDN User Part } \\ \text { IUT } & \text { Implementation Under Tester }\end{array}$


ITU

LT

MAP

MoU/CONIG

MTC

MSC

MTP

OMC

PCO

PSTN

PTC

SCCP

SMS-GMSC

SMS-IWMSC

SS7

SUT

TCAP

TTCN

UT

VLR
International Telecommunication Union

Lower Tester

Mobile Application Protocol

Memorandum of Understanding/Conformance Network Interface Group

Master Tester Component

Mobile Switching Centre

Message Transfer Part

Operation and Maintenance Center

Point of Control and Observation

Public Service Telecommunication Network

Parallel Test Component

Signalling Connection Control Part (SS7 protocol)

Short Message Gateway MSC : Gateway MSC dedicated to the routing of incoming short message

Short Message Interworking MSC : dedicated to the routing of outgoing short message

ITU Signalling System number 7

System Under Test

Transaction Capability Part (SS7 protocol)

Tree and Tabular Combined Notation

Upper Tester

Visited Location Register 